\title{
Efeitos da Reeducação Postural Global em escolares com escoliose
}

\author{
Global Posture Reeducation effects in students with scoliosis
}

Pollyana Coelho Vieira Toledo ${ }^{1}$, Danielli Braga de Mello², Maria Erivania Araújo ${ }^{1}$, Rejane Daoud ${ }^{3}$, Estélio Henrique Martin Dantas ${ }^{4}$

Estudo desenvolvido na Escola Estadual Rotary Club - Governador Valadares (MG), Brasil.

${ }^{1}$ Mestre; Professora colaboradora do Laboratório de Biociências da Motricidade Humana da Universidade Federal do Estado do Rio de Janeiro (UNIRIO) - Rio de Janeiro (RJ), Brasil.

2 Professor Doutor adjunto I da Escola de Educação Física do Exército (EsEFEx) - Rio de Janeiro (RJ), Brasil.

3 Professora colaboradora do Laboratório de Biociências da Motricidade Humana da UNIRIO Rio de Janeiro (RJ), Brasil.

${ }^{4}$ Professor Doutor do Programa de Pós-graduação em Enfermagem e Biociências Humana da UNIRIO Rio de Janeiro (RJ), Brasil.

\section{ENDEREÇO PARA CORRESPONDÊNCIA}

Pollyana Coelho Vieira Toledo Rua Olegario Maciel, 569 - apto 801 - Esplanada - CEP: 35010200 - Governador Valadares (MG), Brasil - E-mail: pollyanacoelhogv@ hotmail.com

\section{APRESENTAÇÃO}

nov. 2010

ACEITO PARA PUBLICAÇÃO

ago. 2011

FONTE DE FINANCIAMENTO nenhuma

\section{CONFLITO DE INTERESSE}

nada a declarar

Aprovação do Comitê de Ética UCB/VREPGPE/COMEP/PROCIMH com parecer favorável 0109/2008
RESUMO: O objetivo deste estudo foi analisar o efeito do método da Reeducação Postural Global (RPG) em escolares com diagnóstico de escoliose torácica não estrutural (ETNE). Os escolares com indicativo de ETNE ao exame postural e teste de Adams negativo foram encaminhados ao exame radiográfico para comprovação diagnóstica. Foram selecionados 20 participantes (11 meninos e 9 meninas, com $10 \pm 3$ anos), divididos randomicamente em dois grupos homogêneos: o que realizou o RPG (GRPG) durante 12 semanas com duração de 25 a 30 minutos cada sessão, de acordo com o que aguentou permanecer na postura; e o grupo controle (GC), sem intervenção. Após três meses, os dois grupos repetiram a avaliação postural e o exame radiográfico. Para avaliação das estatísticas, foi utilizada análise de variância (ANOVA) univariada, com medidas repetidas, seguida do Post Hoc de Tukey para identificar as possíveis diferenças intra e intergrupos. O valor de $\alpha$ foi de 0,05 . O GRPG apresentou redução significativa no ângulo de Cobb na comparação intragrupo $(\Delta \%=-35,100 ; p=0,009)$, mas o GC não $(\Delta \%=9,520 ; p=0,789)$. Pode-se concluir que escolares submetidos ao método da RPG apresentaram melhora do quadro de escoliose torácica não estrutural.

Descritores: postura; escoliose; menores de idade.

ABSTRACT: The aim of this study was to analyze the effect of Global Reeducation Postural (RPG) method in scholars diagnosed with thoracic nonstructural scoliosis (ETNE). Scholars with indicative of ETNE to postural exam and Adams test negative were directed to radiographic exam to diagnostic. Twenty participants were selected ( 11 boys and 9 girls, 10 \pm 3 years old) and randomly divided in two homogeneous groups: group treated by RPG (GRPG) and control group (CG). The GRPG was submitted a RPG treatment during 12 weeks in sessions from 25 to 30 minutes in agreement with each student tolerance at posture. The CG didn't suffer intervention. After three months, both groups repeated the postural and radiographic exam. For statistics, it was used ANOVA univariated with repeated measures, followed by Tukey Post Hoc Test to identify the possible differences intra and intergroups. The $\alpha$ value used was 0.05 . The GRPG present significant reduction at Cobb angle between pre and posttest $(\Delta \%=-35.100 ; p=0.009)$, but the CG didn't show $(\Delta \%=9.520 ; p=0.789)$. It was concluded that scholars submitted to RPG method presented improvement in thoracic nonstructural scoliosis.

KEYWords: posture; scoliosis; minors. 


\section{INTRODUCÃO}

A escoliose é uma deformidade que afeta a forma tridimensional da coluna vertebral e pode ocorrer em qualquer fase da vida, mas principalmente a partir dos dez anos de idade ${ }^{1}$, com progressão associada ao estirão do crescimento. A prevalência da escoliose no escolar varia de 1 a $3 \%$ da população. Assim, a detecção precoce desta patologia aumenta em até três vezes o número de pacientes tratados, diminuindo o percentual dos que necessitam de cirurgia ${ }^{2}$.

É clinicamente importante que se diferencie a escoliose estrutural da escoliose não estrutural. A não estrutural pode ser causada por maus hábitos posturais e discrepância de membro, entre outros; já a estrutural apresenta rotação das vértebras e a coluna torna-se rígida devido à anormalidade nesta estrutura, formando a giba.

A giba, portanto, é a rotação da vértebra para o lado da convexidade da curva e a sua manifestação clínica juntamente com a deformidade das costelas na coluna torácica ou dos processos espinhosos na coluna lombar. Em escolares, a escoliose não estrutural é comum, necessitando de tratamento ${ }^{3-6}$.

A fisioterapia conta com diversos métodos de tratamento: uso de colete, iso-stretching, osteopatia ${ }^{7}$, método $k$ lapp $^{8}$, pilates $^{9}$ e a Reeducação Postural Global $(\mathrm{RPG})^{4,10}$. O último melhora o corpo, corrige a morfologia, suprime a rigidez e libera as articulações, tratando a desarmonia levando em consideração as necessidades individuais de cada ser ${ }^{11}$, o que é fundamental para a correção da escoliose não estrutural, por se tratar da fase de crescimento e porque é nesse período que se restabelece a fisiologia normal da coluna ${ }^{12}$.

Rosário et al. ${ }^{13}$ esclarece que a RPG promove o alongamento global das cadeias musculares e preconiza utilização de posturas específicas para o alongamento dos músculos. As cadeias musculares são constituídas por músculos gravitacionais que trabalham de forma sinérgica dentro da mesma cadeia ${ }^{10}$.

A RPG parte do pressuposto que um músculo encurtado cria compensações em músculos próximos ou distantes ${ }^{10}$.
Desta forma, melhora as alterações biomecânicas encontradas com base nas ações fisiológicas, atuando não só no sistema músculo-esquelético propriamente dito, mas também no nervoso ${ }^{14}$, responsável pelo armazenamento de informações, modificando, assim, a consciência corporal.

Marques $^{12}$ apresenta um caso de escoliose torácica estrutural tratada com a RPG, apresentando diminuição da curva. Em outro estudo, Fregonesi et al. ${ }^{15}$ submeteram uma adolescente com diagnóstico de escoliose estrutural ao tratamento da RPG e a mesma não reduziu a angulação da escoliose.

O presente trabalho tem como objetivo avaliar os efeitos do método da Reeducação Postural Global no ângulo de Cobb de escolares do $5^{\circ}$ ano com diagnóstico de escoliose torácica não estrutural.

\section{MATERIAL E MÉTODOS}

Esta pesquisa adotou o modelo estudo experimental e contou com a participação de 20 escolares (11 meninos e nove meninas) do $5^{\circ}$ ano do ensino fundamental da Escola Estadual Rotary Club, divididos aleatoriamente em dois grupos: RPG (GRPG; $n=10$ ) e controle (GC; $n=10)$. O critério de inclusão foi ter escoliose funcional ${ }^{9,17}$; os escolares que apresentaram indicativo de escoliose não estrutural torácica em " $\mathrm{C}$ " ao exame postural, e teste de Adams negativo, foram encaminhados ao exame de raio $\mathrm{X}$. Foi motivo de exclusão a presença de escoliose estrutural. Os responsáveis pelas crianças participantes do estudo assinaram o Termo de Consentimento Livre e Esclarecido.

O presente estudo está de acordo com as normas éticas para a realização de pesquisa em seres humanos, r196/96 do Conselho Nacional de Saúde de 10/10/1996 (BRASIL, 1996) e da resolução de Helsinki (WMA, 2008) e teve seu projeto devidamente aprovado pelo Comitê de Ética em Pesquisa da Universidade Castelo Branco sob o número 0109/2008.

O exame radiográfico foi utilizado para medir o ângulo da escoliose pelo método de Cobb, que se baseia em radiografias e é referência para medição da angulação frontal da escoliose. A medida é feita traçando-se uma linha perpendicular à margem superior da vértebra que mais se inclina em direção à concavidade, e outra na borda inferior da vértebra, com maior angulação na direção da concavidade. A interseção das duas determina o ângulo do desvio na coluna $^{16}$. Já o índice de Risser, essencial no manejo de escolares com escoliose ${ }^{17}$, corresponde à presença de calcificação na apófise ilíaca, que mede a progressiva ossificação associada ao fechamento do crescimento ósseo ${ }^{18}$, apresentando classificação de 1 a $5^{17}$.

A radiografia antero-posterior (AP) foi realizada no período da noite por um técnico em radiologia com equipamento de raio $X$ da marca VMI Pulsar Plus $^{\circledR}, 800$ mA (Miliampére) com 125 kv (Kiloelétron volt) e console de comando digital. $A$ incidência de raio $X$ no tórax é calculada em 300 mA, 5 mAS, 75 KV, espessura (tórax) de $25 \mathrm{~cm}$. Para o exame, o escolar permaneceu em posição ortostática. A radiografia envolveu pelve, coluna lombar, coluna torácica e parte da coluna cervical com incidência AP.

Uma fisioterapeuta, mediante a avaliação postural e exame de raio X, determinou o grau da escoliose não estrutural. Após a avaliação inicial, o GRPG foi submetido ao tratamento durante três meses (12 semanas), duas vezes por semana, respeitando o tempo limite de cada escolar durante as posturas ${ }^{19}$, que variou de 25 a 30 minutos, mantendo intervalo de 2 minutos entre cada uma delas. A evolução das posturas foi de acordo com o controle da respiração, a capacidade de manter o alinhamento e correções necessárias, dependendo basicamente das condições apresentadas por cada escolar ${ }^{20}$.

Foram utilizadas as posturas "rã no chão" (Figura 1) e "rã no ar" (Figura 2), que permitem melhor abordagem durante as mesmas por serem em decúbito dorsal e não terem carga. Elas fazem, simultaneamente, o trabalho isométrico dos músculos estáticos, sempre com pompagem articular na coluna cervical e coluna lombo-sacra, sendo progressiva, cada vez mais global $^{21}$, ou seja, não é tratada só a coluna do escolar e sim o corpo como um todo. 
Para a postura "rã no chão", que consiste no tronco e membros inferiores em extensão, os escolares foram posicionados em decúbito dorsal com os braços ao longo do corpo, radiulnares em supinação, membros inferiores em abdução com rotação lateral, com flexão de quadril e joelhos até a completa aposição das plantas dos pés ${ }^{22}$. A progressão da postura em abertura de ângulo consiste na extensão e adução dos membros inferiores e abertura dos membros superiores ${ }^{21}$.

Já a "rã no ar" consiste no tronco e membros inferiores estarem em ângulo de $90^{\circ}$ graus. Os escolares permaneceram posicionados em decúbito dorsal, porém com os ombros em abdução a aproximadamente $45^{\circ}$, com a articulação radiulnar em supinação, membros inferiores em abdução e suspensos pelo calcâneo (por uma corda fixada na parede) com as talocrurais em dorsiflexão e paralelos ao teto $^{22}$. A progressão da postura em fechamento de ângulo consiste na flexão e adução dos membros inferiores.

As posturas da RPG dependem da concentração e participação do paciente em seguir as orientações do fisioterapeuta e realizar as correções necessárias durante o decorrer da postura, que proporciona o alongamento muscular ativo e contrações isométricas cada vez mais excêntricas dos músculos encurtados ${ }^{23}$.

No transcorrer das posturas, foram realizadas pompagens pelo fisioterapeuta, visando o alinhamento das curvaturas dorsal e cervical da coluna vertebral ${ }^{24}$.

O GRPG foi orientado sobre como carregar a mochila, e a cada sessão da reeducação foi possível observar a melhora da conscientização corporal e também da postura. Já o GC não realizou tratamento e, a exemplo do realizado com o GRPG, também foi orientado quanto à melhor forma de carregar a mochila durante os três meses, entre o primeiro e o último raio $X$.

Após o trimestre, o GRPG e o GC passaram novamente pela avaliação postural e exame de raio $X$ para análise dos resultados. Todo o procedimento de aplicação das avaliações diagnóstica e somativa, bem como dos exercícios, foi realizado pela mesma fisioterapeuta, que tem certificado de qualificação do método RPG no Brasil.

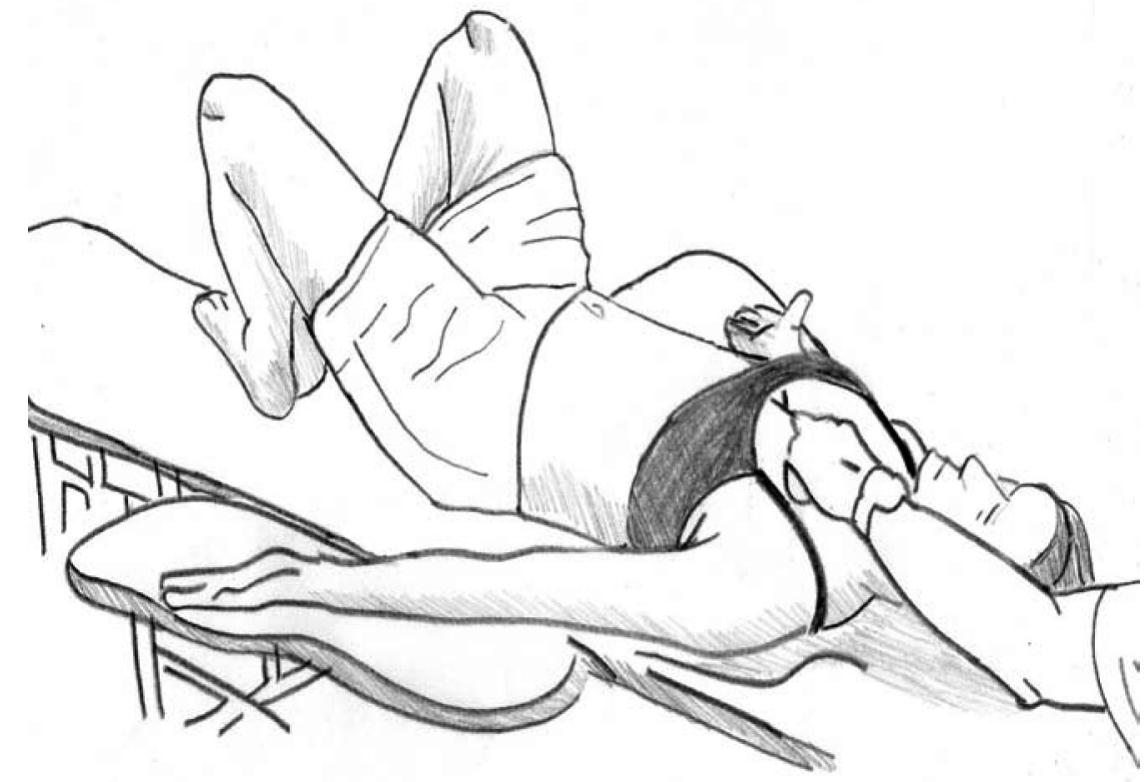

Figura 1. Postura rã no chão

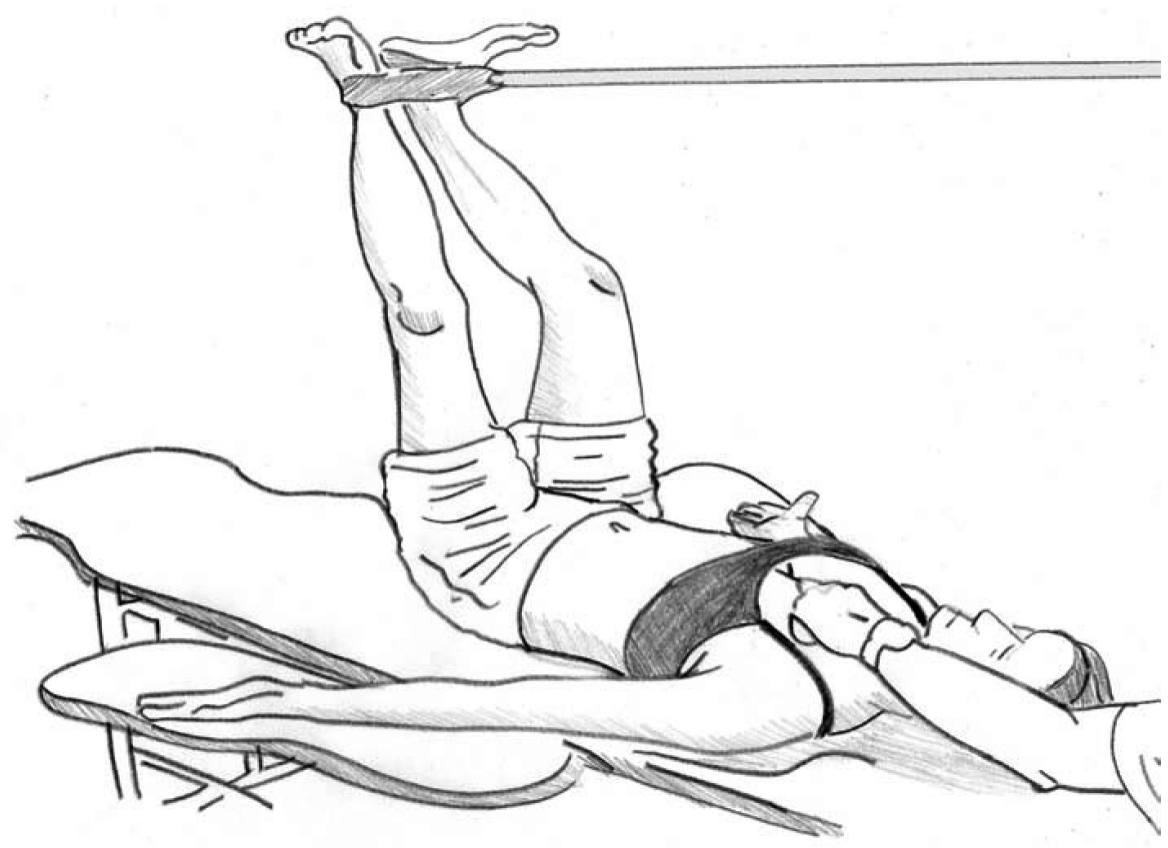

Figura 2. Postura rã no ar

Para a análise dos resultados, em um primeiro momento foram utilizadas técnicas de estatística descritiva, por meio de média, desvio padrão, erro padrão, valores mínimos e máximos. A normalidade e homogeneidade de variância dos dados da amostra foram verificadas com o emprego do teste de Shapiro-Wilk e Levene, respectivamente. Empregou-se a análise de variância
(ANOVA) univariada com medidas repetidas, seguida do Post Hoc de Tukey para identificar as possíveis diferenças intra e intergrupos para a variável ângulo de Cobb. O valor de $\alpha$ utilizado foi de 0,05 .

Os escolares GRPG (4 meninas e 6 meninos) e GC ( 5 meninas e 5 meninos) apresentam a mesma idade (10 anos) e faziam parte da mesma turma do $5^{\circ}$ ano 


\section{Incidência de Escoliose}

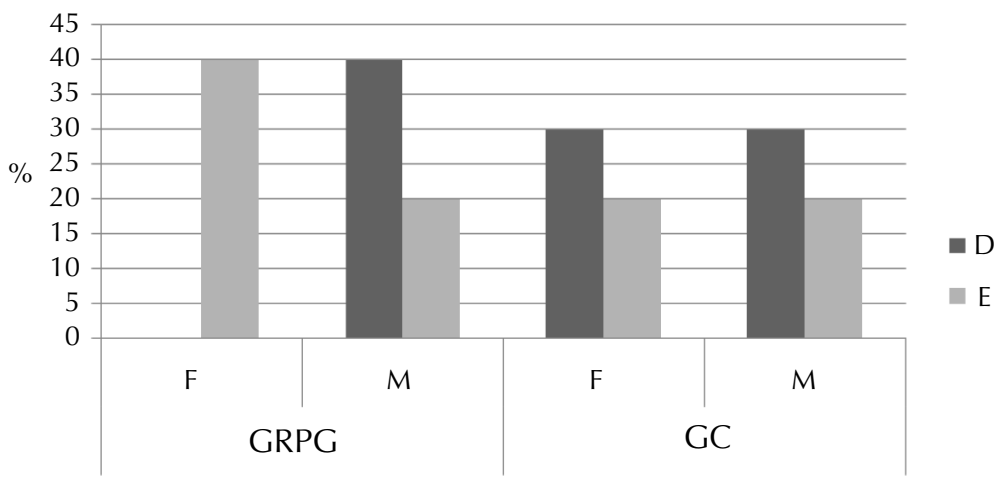

Convexidade da escoliose

GRPG: grupo RPG; GC: grupo Controle; D: direita; E: esquerda; F: sexo feminino; $M:$ sexo masculino.

Figura 3. Incidência da convexidade da escoliose não estrutural

Tabela 1. Análise intra e intergrupos, entre os grupos GRPG e GC do ângulo de Cobb (graus)

\begin{tabular}{cccccccc}
\hline & & Média & $\begin{array}{c}\text { Desvio } \\
\text { padrão }\end{array}$ & $\begin{array}{c}\text { Valor } \\
\text { mínimo }\end{array}$ & $\begin{array}{c}\text { Valor } \\
\text { Máximo }\end{array}$ & $\Delta \%$ & $\begin{array}{c}\text { Valor p } \\
\text { intragrupo }\end{array}$ \\
\hline GRPG & Pré & 15,10 & 2,51 & 12,00 & 20,00 & $-35,10$ & $0,009^{\mathrm{a}}$ \\
& Pós & $9,80^{\mathrm{b}}$ & 2,90 & 6,00 & 14,00 & & \\
GC & Pré & 14,70 & 3,77 & 10,00 & 20,00 & 9,52 & $0,789^{\mathrm{a}}$ \\
& Pós & $16,10^{\mathrm{b}}$ & 3,75 & 10,00 & 22,00 & & \\
\hline
\end{tabular}

GRPG: grupo RPG; GC: grupo Controle; $\Delta \%$ : diferença percentual; $p<0,05$. $\mathrm{a}$ : Diferença significativa intragrupos; $b$ : diferença significativa intergrupos no pós-teste $(p=0,001)$

Tabela 2. Distribuição de frequência da convexidade da escoliose e índice de Risser entre os grupos

\begin{tabular}{lccccc}
\hline & & \multicolumn{2}{c}{ GRPG } & \multicolumn{3}{c}{ GC } \\
\cline { 2 - 6 } & & $M$ & $F$ & $M$ & F \\
\hline Escoliose & $\mathrm{D}$ & 5 & 1 & 3 & 3 \\
\multirow{2}{*}{ Risser1 } & $\mathrm{E}$ & 1 & 3 & 2 & 2 \\
& $\mathrm{D}$ & 5 & 1 & 3 & 3 \\
Risser2 & $\mathrm{E}$ & 0 & 3 & 2 & 1 \\
& $\mathrm{D}$ & 0 & 0 & 0 & 0 \\
\hline Total & $\mathrm{E}$ & 1 & 0 & 0 & 1 \\
\hline
\end{tabular}

GRPG: grupo RPG; GC: grupo Controle; D: direita; E: esquerda; F: sexo feminino; $M$ : sexo masculino

do ensino fundamental, estando submetidos à mesma sobrecarga de material e rotina escolar.

A incidência da convexidade da escoliose não estrutural em ambos os grupos pode ser visualizada na Figura 3.
A Tabela 1, a seguir, apresenta o grau de curvatura da escoliose por meio do ângulo de Cobb no GRPG e GC.

Observou-se população com distribuição normal antes da intervenção. Ao comparar os valores médios do ângulo de Cobb apresentados antes e após o período de intervenção, observou-se que o GRPG demonstrou redução significativa $(p=0,050)$ no grau de curvatura da escoliose após a realização da RPG, enquanto o GC registrou aumento não significativo $(p=0,789)$ no grau de curvatura, o que indica agravamento no quadro de escoliose.

A amostra analisada (GRPG e GC) apresenta o índice de Risser 1(corresponde à presença de ossificação ocupando até $25 \%$ lateral da extensão total da epífise e o índice de Risser 2 (apresenta ossificação ocupando de 26 até 50\% lateral da extensão total da epífise $)^{25}$. A distribuição de frequência da convexidade da escoliose e o índice de Risser entre os grupos podem ser observados na Tabela 2.

DISCUSSÃO

Pouco se sabe sobre a progressão da curva da escoliose, mas vários fatores afetam este processo. Enquanto flexível ou não estruturada, se torna mais fácil sua correção e/ou a estabilização, ou seja, ao final do crescimento, sua probabilidade de correção é menor ${ }^{8}$.

O objetivo deste estudo foi avaliar os efeitos do método da Reeducação Postural Global no ângulo de Cobb de escolares com diagnóstico de escoliose não estrutural. O método da RPG foi escolhido porque alonga número grande de músculos de uma única vez, não permitindo que haja compensações. Já o alongamento segmentar alonga um músculo ou grupo muscular específico, contudo, pode se tornar menos eficiente devido às compensações secundárias que podem ocorrer na respectiva cadeia ${ }^{10}$.

Esta pesquisa foi realizada com escolares de dez anos de idade devido ao início do estirão de crescimento. O tratamento fisioterapêutico com a RPG foi escolhido porque, além de considerar o indivíduo de forma global, tem como objetivo o conhecimento do próprio corpo e, assim, o aperfeiçoamento das reações de endireitamento. Martell ${ }^{6}$, em seu estudo, descreve que a escoliose idiopática tem incidência em escolares de 10 a 16 anos, os quais são considerados população de risco devido à idade, pontuando ainda a importância da autoimagem e de que a 
postura adequada na infância é importante para prevenir problemas futuros.

Curto et al. ${ }^{26}$ avaliaram duas adolescentes de dez anos. Elas não foram tratadas durante o período da pesquisa. A primeira apresentou curva lombar com ângulo de $17^{\circ}$ cobb e curva torácica de $14^{\circ}$ cobb. Após um ano, a curva evoluiu: a lombar passou a ser $24^{\circ}$ cobb e a torácica $23^{\circ} \mathrm{cobb}$. A segunda adolescente apresentava escoliose tóraco-lombar de $18^{\circ}$ cobb, que após um ano evoluiu para $40^{\circ}$ cobb.

Os resultados obtidos no presente estudo demonstram que o GRPG apresentou diminuição significativa no ângulo da escoliose, enquanto que o GC registrou aumento. Uma justificativa para a diminuição da curva é que a RPG atua do centro para a extremidade do corpo, ou seja, da coluna para os membros, utilizando o alongamento muscular ativo envolvendo, em conjunto, os músculos estáticos antigravitários, os rotadores internos e os inspiratórios ${ }^{27}$, restabelecendo o equilíbrio muscular.

Neste sentido, seguem outros métodos que avaliaram o resultado do tratamento da escoliose, como no estudo de Molina e Camargo ${ }^{4}$ que trataram 9 crianças de 9 a 15 anos com postura de alongamento isotônico excêntrico e obtiveram como resultado diminuição no ângulo da escoliose, avaliada pelo método de Cobb. Já Monte-Raso et al. ${ }^{28}$ utilizaram o iso-stretching para melhorar alterações posturais como a escoliose, com 12 voluntários. A autora relata que o método não foi eficaz com o tratamento das assimetrias.

O resultado da análise radiológica em relação ao ângulo de Cobb pode ser comparado ao estudo realizado por Oliveira e Souza ${ }^{7}$, que aplicaram o método do iso-stretching e manipulações osteopáticas em seu estudo com a escoliose, chegando ao resultado de $66,7 \%$ de redução no ângulo de Cobb e $16,7 \%$ dos pacientes com curvatura estabilizada. No presente estudo, o GRPG obteve diminuição no ângulo da escoliose em todos os escolares.

A escoliose e seu tratamento apresentam grande impacto na qualidade de vida dos escolares, ocasionando diferenças culturais em sua percepção ${ }^{29}$.

No estudo de Marques ${ }^{12}$ e Fregonesi ${ }^{15}$, o tratamento da escoliose estrutural com a RPG alcançou resultados diferentes. O primeiro autor conseguiu diminuir em $10^{\circ}$ o ângulo. Já o outro, mesmo submetendo o escolar a um ano de tratamento semanal, não obteve melhora na curvatura, porém conseguiu estabilizar a curva. Neste estudo, o GRPG registrou diminuição média de 35,10\% no ângulo da escoliose, e o GC, aumento de 9,52\%. Rosário et al. ${ }^{10}$ esclarece que a RPG se mostra eficiente no tratamento do desvio postural, no entanto, a literatura ainda é escassa $^{12}$.

No GC o aumento encontrado na escoliose pode estar relacionado às posturas inadequadas durante a fase do estirão do crescimento ${ }^{8}$.

Deste modo, a escoliose não estrutural tratada com a RPG diminui a curva na coluna e/ou melhora as alterações posturais, viabilizando a capacidade do escolar em perceber o próprio corpo $^{22}$. Neste caso, os músculos trabalham em conjunto tanto para sua estática como para sua dinâmica e o sistema nervoso central age de forma tridimensional. Portanto, qualquer alteração postural causará retração de suas cadeias, o que levará a desalinhamento ósseo. Souchard ${ }^{22}$ esclarece que cada movimento que realizamos necessita de ações de toda a estrutura óssea, muscular e ligamentar para manter o equilíbrio e perceber o próprio corpo é fundamental ${ }^{14}$.

Assim, os exercícios terapêuticos têm se mostrado uma eficiente ferramenta para a melhora da escoliose e/ou sua estabilização7. A RPG resgata o equilíbrio músculo-esquelético por meio do alongamento, da contração muscular, da consciência corporal e da correção postural. Desse modo, os princípios básicos da RPG na escoliose foram melhorar a retração dos músculos da estática; corrigir, por intermédio da tração, a acentuação das curvas; estirar, de forma global, os músculos da estática e liberar o bloqueio respiratório ${ }^{30}$.

Alguns estudos a respeito da RPG demonstraram a eficácia e as limitações deste método em diversas patologias, inclusive na escoliose. Contudo, a prevenção, ou a atenção primária, se faz necessária, e a partir deste pensamento a escoliose não estrutural se torna prioridade.

\section{CONCLUSÃO}

Pode-se concluir que escolares submetidos ao método da Reeducação Postural Global apresentaram melhora do quadro de escoliose torácica não estrutural.Diante disto, o presente estudo recomenda a RPG para o tratamento, contudo, se faz necessário avaliar o escolar de seis em seis meses para observar o desenvolvimento de sua coluna vertebral durante toda a fase de estirão do crescimento. Deve-se enfatizar, ainda, que serão necessárias pesquisas com um período de tempo maior para avaliar o protocolo de tratamento, uma vez que estudos científicos a esse respeito são escassos.

\section{REFERÊNCIAS}

1. Zurita OF, Moreno LC, Ruiz RL, Martínez MA, Zurita OA, Castro SAM. Screening of scoliosis in a school population of 8 to 12 years in the province of Granada (Spain). An Pediatr. 2008;69(4):342-50.

2. Dohnert MB, Tomasi E. Validade da fotogrametria computadorizada na detecção de escoliose idiopática adolescente . Rev Bras Fisioter. 2008;12(4):290-7.
3. Harrison DE, Betz JW, Cailliet R, Colloca CJ, Harrison DD, Haas JW, et al. Radiographic pseudoscoliosis in healthy male subjects following voluntary lateral translation (side glide) of the thoracic spine. Arch Phys Med Rehabil. 2006;87(1):117-22.

4. Molina Al, Camargo OP. O tratamento da criança com escoliose por alongamento muscular. Fisioter Bras. 2003;4(5):369-72. 


\section{Referências (cont.)}

5. Leal JS, Leal MCPS, Gomes CER, Guimarães MDC. Inquérito epidemiológico sobre escoliose idiopática do adolescente. Rev Bras Ortop. 2006;41(8):309-19.

6. Martelli RC, Traebert J. Estudo descritivo das alterações posturais de coluna vertebral em escolares de 10 a 16 anos de idade: Tangará-SC, 2004. Rev Bras Epidemiol. 2006;9(1):87-93.

7. Oliveira AP, Souza DE. Tratamento Fisioterapêutico em Escoliose através das Técnicas de Iso-Stretching e Manipulações Osteopáticas. Terapia Manual. 2004;2:104-13.

8. Iunes DH, Cecílio MBB, Dozza MA, Almeida PR. Análise quantitativa do tratamento da escoliose idiopática com o método Klapp por meio de biofotogrametria computadorizada. Rev Bras Fisioter. 2010;14(2):133-40.

9. Araujo MEA, Silva EB, Vieira PC, Cader SA, Mello DB, Dantas EHM. Redução da dor crônica associada à escoliose não estrutural, em universitárias submetidas ao método Pilates. Motriz: Ver Educ Fis. 2010;16(4):958-66.

10. Rosário JLP, Souza A, Cabral CMN, João SMA, Marques AP. Reeducação Postural Global e alongamento estático segmentar na melhora da flexibilidade, força muscular e amplitude de movimento: um estudo comparativo. Fisioter Pesq. 2008;15(1):12-8.

11. Lima PG, Costa MAM. Variações dolorosas encontradas em pacientes escolióticos submetidos a técnica da Reeducação Postural Global (RPG). Publicar Revista Digital Cadernos de Saúde. FAL. 2007;1:262-272.

12. Marques AP. Escoliose tratada com Reeducação Postural Global. Rev Fisioter. 1996;3(1/2):65-8.

13. Rosario JL, Marques AP, Maluf AS. Aspectos clínicos do alongamento: uma revisão da literatura. Rev Bras Fisioter. 2004;8(1):83-8.

14. Teodori RM, Guirro ECO, Santos RM. Distribuição da pressão plantar e localização do centro de pressão após intervenção pelo método de Reeducação Postural Global: um estudo de caso. Fisioter Mov. 2005;8:27-35.

15. Fregonesi CEPT, Valsechi CM, Masselli MR, Faria CRS, Ferreira DMA. Um ano de evolução da escoliose com RPG. Fisioter Bras. 2007;8(2):140-2.

16. Baraúna MA, Canto RST, Sanchez HM, Bustamante JCF, Ventura-Silva RA, Malusá S. Validade e confiabilidade intra-indivíduo do cifolordômetro na avaliação da convexidade torácica. Rev Bras Fisioter. 2005;9(3): 319-25.

17. Thaler M, Kaufmann G, Steingruber I, Mayr E, Liebensteiner M, Bach C. Radiographic versus ultrasound evaluation of the Risser Grade in adolescent idiopathic scoliosis: a prospective study of 46 patients. Eur Spine J. 2008;17(9):1251-5.
18. Döhnert MB, Tomasi E. Validade da fotogrametria computadorizada na detecção de escoliose idiopática adolescente. Rev Bras Fisioter. 2008;12(4):290-7.

19. Moreira CMC, Soares DRL. Análise da efetividade da Reeducação Postural Global na protusão do ombro após a alta terapêutica. Fisioter Mov. 2007;20(1):93-9.

20. Moreno MA, Catai AM, Teodori RM, Borges BLA, Zuttin RS, Silva E. Adaptações do sistema respiratório referentes a função pulmonar em resposta a um programa de alongamento muscular pelo método da Reeducação Postural Global. Fisioter Pesq. 2009;16(1):11-5

21. Gomes BM, Nardoni GCG, Lopes PG, Godoy E. O efeito da técnica de reeducação postural global em um paciente com hemiparesia após acidente vascular encefálico. Acta Fisiátrica. 2006;13(2):103-8.

22. Souchard PE. O papel biomecânico dos músculos espinhais: sua implicação na escoliose. Fisioter Bras. 2001;2(6):369-78.

23. Mota YL, Barreto SL, Bin PR, Simões HG, Campbel CSG. Respostas cardiovasculares durante a postura sentada da Reeducação Postural Global (RPG). Rev Bras Fisioter. 2008;12(3):161-8.

24. Silva E, Moreno MA, Catai AM, Teodori RM, Borges BLA, Zuttin RS, et al. Avaliação da influência do método de Reeducação Postural Global (RPG) sobre as respostas cardiorespiratórias. In: V Congresso de Pesquisa, Piracicaba, 23-28 outubro 2007. Piracicaba: Mostra Acadêmica Unimep; 2007. p.1-5.

25. Thaler M, Kaufmann G, Steingruber I, Mayr E, Liebensteiner M, Bach C. Radiographic versus ultrasound evaluation of the Risser Grade in adolescent idiopathic scoliosis: a prospective study of 46 patients. Eur Spine J. 2008;17(9):1251-5.

26. Curto DD, Ueta RHS, Wajchenberg M, Filho DEM, Puertas EB. Variações na apresentação fenotípica da escoliose idiopática do adolescente. Coluna/Columna. 2010;9(1):19-23.

27. Malgorzata L. Changes in the anterior-posterior spinal curvatures in children aged 6-7 years. Physical Education and Sport. 2008;52:1-4.

28. Monte-Raso VV, Ferreira PA, Carvalho MS, Rodrigues JG, Martins CC, lunes DH. Efeito da técnica isostretching no equilíbrio postural. Fisioter Pesq. 2009;16(2):137-42.

29. Rosanova GCL, Gabriel BS, Camarini PMF, Gianini PES, Coelho DM, Oliveira AS. Validade concorrente a versão brasileira da SRS-22r com o Br-SF-36. Rev Bras Fisioter. 2010;14(2):121-6.

30. Souchard PE, Ollier M. As escolioses - Seu tratamento fisioterapêutico e ortopédico. São Paulo: E realizações; 2001. p.66-145. 